\title{
Softened Symmetry Breaking in Composite Higgs Models
}

\author{
Simone Blasi®* and Florian Goertz $\oplus^{\dagger}$ \\ Max-Planck-Institut für Kernphysik Saupfercheckweg 1, 69117 Heidelberg, Germany
}

(Received 30 July 2019; revised manuscript received 17 September 2019; published 26 November 2019)

\begin{abstract}
We propose a new way of breaking the Goldstone symmetry in composite Higgs models, restoring the global symmetry in the mixings between the elementary and composite fermions by completing the former to full representations of this symmetry. The Goldstone symmetry is in turn broken softly by vectorlike mass terms in the elementary sector only. The resulting softened explicit breaking allows for a light Higgs boson, as found at the LHC, and a heavy top quark, without the need of light top partners around the Goldstone scale $f \sim \mathrm{TeV} \ll m_{\text {comp }}$, which remain elusive at the LHC, while we recover the standard scenario in the limit of infinite vectorlike masses.
\end{abstract}

DOI: 10.1103/PhysRevLett.123.221801

Introduction.-Composite Higgs $(\mathrm{CH})$ models offer a promising means to solve the hierarchy problem-one of the biggest puzzles in fundamental physics - since the Higgs boson is no longer a fundamental scalar but rather a bound state of a new strong interaction, which can be resolved above the $\mathrm{TeV}$ scale, and its mass is thus saturated in the IR [1-3]. Moreover, the conventional assumption of the Higgs boson being a (pseudo-) Goldstone boson of a spontaneously broken global symmetry $[S O(5) \rightarrow S O(4)$ in minimal models] provides an intriguing reasoning for its lightness compared to other new states that have not been discovered so far. In the same framework, partially composite fermions (elementary fields mixing linearly with the composite sector of bound states) can also explain the hierarchical structure of fermion masses and mixings [4-7] and provide a dynamical origin for electroweak symmetry breaking (EWSB), mostly triggered by the large topquark compositeness. The latter explicitly breaks the global symmetry, since the standard model (SM) fermions do not fill complete representations of the global symmetry that could couple to the composite sector in an invariant way, and thereby induces a potential for the Goldstone Higgs boson, intertwining flavor and EWSB.

Minimal models are, however, in increasing tension with the absence of light top partners at the LHC, which are required to keep the Higgs boson light by reducing the Goldstone-symmetry breaking [7-11], threatening the viability of explicit $\mathrm{CH}$ incarnations (see, e.g., Ref. [12]). While one possibility to avoid this is the

Published by the American Physical Society under the terms of the Creative Commons Attribution 4.0 International license. Further distribution of this work must maintain attribution to the author(s) and the published article's title, journal citation, and DOI. Funded by SCOAP. inclusion of a realistic lepton sector [13], which can have a non-negligible impact with interesting consequences for flavor physics [12-16] (see also Ref. [17] for a solution via an enlarged quark sector), here we want to explore an orthogonal solution, changing in fact the nature of the explicit Goldstone symmetry breaking.

Indeed, the latter could be significantly reduced if the SM fermions would be uplifted to complete representations of the global symmetry. In that case, their mixing with the composite sector, determining their degree of "compositeness," would no longer violate the global symmetry. However, the symmetry still needs to be broken, but this is now done by introducing vectorlike mass terms for the new elementary fermions, at the same time accounting for the nonobservation of light states other than the SM ones. This will shift the source of explicit breaking to a different sector and thereby corresponds to a fundamentally different approach of breaking the Goldstone symmetry.

The breaking is now "soft," since induced by mass terms in the elementary sector. Contrary to the conventional case, the underlying interactions between the SM fermions and the constituents of the composite states now preserve the global symmetry. In particular, the setup leads to a different parametric structure of the mass of the composite Higgs boson, allowing us to lift the light top partners. The purpose of this Letter is to work out the phenomenological and conceptual consequences of this approach. Here, we focus on the minimal $S O(5) / S O(4)$ composite Higgs scenario [5], but the considerations can easily be extended to different cosets.

Setup.-We start by recalling that in the $\mathrm{MCHM}_{5}$ [7], the left-handed doublet $q_{L}$ and the singlet $t_{R}$ are embedded as

$$
\psi_{L}^{t}=\Delta_{L}^{t \dagger} q_{L} \sim \mathbf{5}_{2 / 3}, \quad \psi_{R}^{t}=\Delta_{R}^{t \dagger} t_{R} \sim \mathbf{5}_{2 / 3},
$$

where the spurions 


$$
\begin{aligned}
\Delta_{L}^{t} & =\frac{1}{\sqrt{2}}\left(\begin{array}{ccccc}
0 & 0 & 1 & -i & 0 \\
1 & i & 0 & 0 & 0
\end{array}\right), \\
\Delta_{R}^{t} & =-i\left(\begin{array}{lllll}
0 & 0 & 0 & 0 & 1
\end{array}\right)
\end{aligned}
$$

parametrize the $S O(5)$ breaking due to the fact that $q_{L}$ and $t_{R}$ do not fill complete $S O(5)$ multiplets.

In the proposed vectorlike extension with soft Goldstone breaking, the sMCHM${ }_{5}$, we uplift $\psi_{L}^{t}$ and $\psi_{R}^{t}$ to full $S O(5)$ representations by introducing two vectorlike elementary $\mathrm{SU}(2)_{L}$ doublets, $w$ and $v$, and a singlet $s$,

$$
\begin{aligned}
& \psi_{L}^{t}=\Delta_{L}^{t \dagger} q_{L}+\Delta_{w}^{\dagger} w_{L}+\Delta_{S}^{\dagger} s_{L}, \\
& \psi_{R}^{t}=\Delta_{R}^{t \dagger} t_{R}+\Delta_{w}^{\dagger} w_{R}+\Delta_{v}^{\dagger} v_{R},
\end{aligned}
$$

where $\Delta_{s}=\Delta_{R}^{t}, \Delta_{v}=\Delta_{L}^{t}$, and

$$
\Delta_{w}=\frac{1}{\sqrt{2}}\left(\begin{array}{ccccc}
1 & -i & 0 & 0 & 0 \\
0 & 0 & 1 & i & 0
\end{array}\right) .
$$

The new fermions $v$ and $s$ have the same quantum numbers as $q_{L}$ and $t_{R}$, respectively, whereas $w$ is an exotic doublet. Since $v, s$, and $w$ are vectorlike, we can write down the mass terms

$$
\begin{aligned}
-\mathcal{L}_{\mathrm{el}}= & m_{s} \bar{s}_{L} s_{R}+m_{v} \bar{v}_{L} v_{R}+m_{w} \bar{w}_{L} w_{R}+\text { H.c. } \\
= & m_{s} \bar{\psi}_{L}^{t} \Delta_{s}^{\dagger} s_{R}+m_{v} \bar{v}_{L} \Delta_{v} \psi_{R}^{t} \\
& +m_{w} \bar{\psi}_{L}^{t} \Delta_{w}^{\dagger} \Delta_{w} \psi_{R}^{t}+\text { H.c. }
\end{aligned}
$$

where the rewriting in the last two lines makes the $\mathrm{SO}(5)$ breaking by the vectorlike masses apparent due to the presence of the $\Delta$ spurions. Two more mixing terms, namely, $\delta_{1} \bar{q}_{L} v_{R}$ and $\delta_{2} \bar{t}_{R} s_{L}$, are allowed by gauge symmetry. However, their impact is small and we shall include them for completeness only when performing numerical scans, and set $\delta_{1}=\delta_{2}=0$ for analytical estimates.

The elementary fields $\psi_{L}^{t}$ and $\psi_{R}^{t}$ mix with the composite resonances as in the $\mathrm{MCHM}_{5}$, i.e., (see, e.g., Refs. [7,18]),

$$
\begin{aligned}
\mathcal{L}_{\text {mixing }}= & -m_{Q} \bar{Q}_{L} Q_{R}-\tilde{m}_{T} \overline{\tilde{T}}_{L} \tilde{T}_{R} \\
& -y_{L} f_{\pi} \bar{\psi}_{L I}^{t}\left(a_{L} U_{I i} Q_{R}^{i}+b_{L} U_{I 5} \tilde{T}_{R}\right) \\
& -y_{R} f_{\pi} \bar{\psi}_{R I}^{t}\left(a_{R} U_{I i} Q_{L}^{i}+b_{R} U_{I 5} \tilde{T}_{L}\right)+\text { H.c. },
\end{aligned}
$$

where only the lightest top partners are kept and $f_{\pi}$ is the Goldstone-Higgs decay constant. The composite resonances $\tilde{\Psi}^{T}=U(Q, \tilde{T})^{T}$ have been decomposed into fourplets and singlets under the unbroken $S O(4)$ subgroup via the CCWZ prescription, with $U$ the Goldstone matrix. As mentioned, this mixing provides no source of explicit $S O(5)$ breaking, which is sequestered to the elementary sector, coming only from $\mathcal{L}_{\text {el }}$ in Eq. (5). This will in particular lead to a different structure of the Higgs potential.
In contrast to the $\mathrm{MCHM}_{5}$, the $\mathrm{SO}(5)$ breaking spurions are now separated from the Goldstone-Higgs matrix $U$, which requires a larger number of mass-mixing insertions in order to generate a potential, as we also explicitly find in the calculation of the Higgs potential below.

In the 5D holographic dual (see Refs. [5-7]), our setup would correspond to choosing the same boundary conditions for all components of the fermionic bulk fiveplets of $S O(5)$ - whose zero modes contain the SM fermionsthereby respecting $S O(5)$ at the first place. The additional zero modes that emerge due to these universal boundary conditions are then lifted via finite $S O(5)$-breaking vectorlike boundary mass terms on the UV brane. The limit of infinite vectorlike masses reproduces the dedicated boundary conditions that remove those zero modes in the conventional approach. This is similar to realizing EWSB via coupling to an IR-localized Higgs sector with a finite vacuum expectation value (VEV), instead of employing Dirichlet boundary conditions to remove the massless modes of the weak gauge fields.

After all, the soft-breaking setup interpolates between a model with no electroweak symmetry breaking (all vectorlike masses set to zero) and the conventional setup where the explicit breaking comes from fermion partial compositeness (all vectorlike masses set to infinity), the intermediate region being the target of our analysis.

Analysis and numerical results. - We perform our quantitative analysis in a two-site implementation of the $\mathrm{sMCHM}_{5}$, where only the first layer of resonances is kept in the spirit of discrete models, see Refs. [11,18]. The two-site model is given explicitly by the Lagrangian (6), with $a_{L}=a_{R}=b_{L}=b_{R}=1$, together with the elementary fields, whose dynamics is specified by Eq. (5).

The Higgs potential, induced by explicit $S O(5)$ breaking, is computed via the Coleman-Weinberg procedure and can be parametrized as

$$
V(h)=\alpha \sin ^{2}\left(h / f_{\pi}\right)+\beta \sin ^{4}\left(h / f_{\pi}\right) .
$$

Within the two-site model, $\alpha$ is logarithmically divergent as in the $\mathrm{MCHM}_{5}$. In our construction, this turns out to be a consequence of the minimal embedding (3) rather than a generic prediction and $\alpha$ can actually be finite in other realizations of soft breaking. We leave this for further investigation.

The divergence in $\alpha$ can be cured by means of standard renormalization, where the renormalization scale is fixed to reproduce the Higgs VEV [8]. The model is, however, still predictive in terms of $\beta$, which is calculable and connects the fermionic spectrum (entering the latter) to the Higgs mass:

$$
m_{h}^{2}=2 \beta / f_{\pi}^{2} \sin ^{2}\left(2 h / f_{\pi}\right),
$$

where here, and in the following, $h$ denotes the background value of the Higgs field. 
In practice, when scanning the parameter space, we use a renormalized Higgs potential for our two-site model in the spirit of Ref. [8],

$$
V(h)=-\frac{N_{c}}{16 \pi^{2}} \sum_{i} m_{i}(h)^{4}\left[\ln m_{i}(h)^{2} / \mu^{2}-3 / 2\right],
$$

where $m_{i}(h)$ denote for the field-dependent fermion masses, and determine $\mu$ once all the other parameters are fixed in order to reproduce the correct Higgs VEV. We also require $\mu$ to be below the cutoff of the effective theory $\Lambda \approx 4 \pi f_{\pi}$.

In the $\mathrm{MCHM}_{5}$, the top quark mixes with the doublet within $Q$ sharing the quantum numbers of $q_{L}$, denoted by $T$, and with $\tilde{T}$, the singlet with $t_{R}$ quantum numbers. In the $\mathrm{sMCHM}_{5}$, there are two corresponding doublets, identified with the two superpositions of $Q$ and the elementary doublet $v$, which we denote by $T_{+}$and $T_{-}$. Similarly, $\tilde{T}$ splits into two states, made out of $\tilde{T}$ and $s$ and referred to as $\tilde{T}_{+}$and $\tilde{T}_{-}$. As a consequence, the expression for the top mass is modified in the $\mathrm{sMCHM}_{5}$, reading

$$
m_{t}^{2}=y_{L}^{2} y_{R}^{2} f_{\pi}^{4} \frac{m_{s}^{2} m_{v}^{2}\left(m_{Q}-\tilde{m}_{T}\right)^{2}}{8 m_{T_{+}}^{2} m_{T_{-}}^{2} m_{\tilde{T}_{+}}^{2} m_{\tilde{T}_{-}}^{2}} \sin ^{2}\left(2 h / f_{\pi}\right) .
$$

When the $s, v$, and $w$ fields are decoupled taking $m_{s, v, w} \rightarrow \infty$, Eq. (10) approaches the result of the $\mathrm{MCHM}_{5}$ [8] (notice that $m_{T_{ \pm}, \tilde{T}_{ \pm}}$also depend on $m_{s, v}$ ).

We regard the vectorlike masses of the different fermion species, $s, v$, and $w$, as independent quantities. To investigate the relation between the Higgs mass and the top partners, we thus first consider the case in which only the singlet $s$ is active below the condensation scale $\Lambda \sim 4 \pi f_{\pi}$, which is computationally transparent, and finally the case in which all the fermion species contribute.

Singlet case.-We work in the limit in which the singlet $s$ is heavier than $y_{L, R} f_{\pi}$ but still active below the condensation scale, whereas $v$ and $w$ are decoupled. This allows for analytical approximations that can be compared with the full numerical result. For notational convenience we introduce the fourplet-to-singlet mass ratio $q \equiv m_{Q} / \tilde{m}_{T}$ and the composite-to-elementary ratio $r \equiv \tilde{m}_{T} / m_{s}$. The $\mathrm{MCHM}_{5}$ is then recovered for $r=0$. In terms of these ratios, the top mass and the coefficient $\beta$ read

$$
\begin{aligned}
m_{t}^{2} & \simeq \frac{y_{L}^{2} y_{R}^{2} f_{\pi}^{4}}{8 m_{Q}^{2}}(q-1)^{2} \sin ^{2}\left(2 h / f_{\pi}\right), \\
\beta\left(r^{2}\right) & \simeq \frac{3 y_{L}^{2} y_{R}^{2} f_{\pi}^{4}(1-q)^{2}}{16 \pi^{2}\left(1-q^{2} r^{2}\right)}\left[\left(r^{2}+1 / q^{2}\right) F\left(q^{2}\right)-2 F\left(r^{2}\right)\right],
\end{aligned}
$$

where $F\left(x^{2}\right)=\left(x^{2} / 1-x^{2}\right) \ln \left(1 / x^{2}\right)$. As we can see, the top mass is independent of $r$ to first approximation. Conversely, the Higgs potential depends on $r$, and, in particular,

$$
\beta\left(r^{2}\right)-\beta(0) \leq 0,
$$

meaning that the singlet $s$ always reduces the amount of explicit breaking, thus leading to a lighter Higgs boson. Combining Eqs. (8) and (11), we can relate the Higgs mass to the spectrum of resonances as

$$
m_{Q}^{2}=\frac{1}{16} y_{L}^{2} y_{R}^{2} f_{\pi}^{6} \frac{(1-q)^{2}}{\beta\left(r^{2}\right)}\left(\frac{m_{h}}{m_{t}}\right)^{2}
$$

In turn, we estimate the mass of the lightest eigenstate of the system, $m_{l}$, via

$$
\begin{aligned}
m_{l}^{2} & \simeq \min \left\{m_{Q}^{2}, \tilde{m}_{T}^{2}, m_{s}^{2}\right\} \\
& =m_{Q}^{2} \times \min \left\{1,1 / q^{2}\right\},
\end{aligned}
$$

where $m_{Q}^{2}$ is given in Eq. (13) and we have used the fact that the configurations with the singlet $s$ as the lightest state are excluded because of a negative quartic, see below.

The value of $m_{l}$ predicted by Eq. (14) is shown in the upper panel of Fig. 1 as a function of $r$ for different values of $q$. The top mass at the scale $f_{\pi} \approx 800 \mathrm{GeV}$ is fixed to
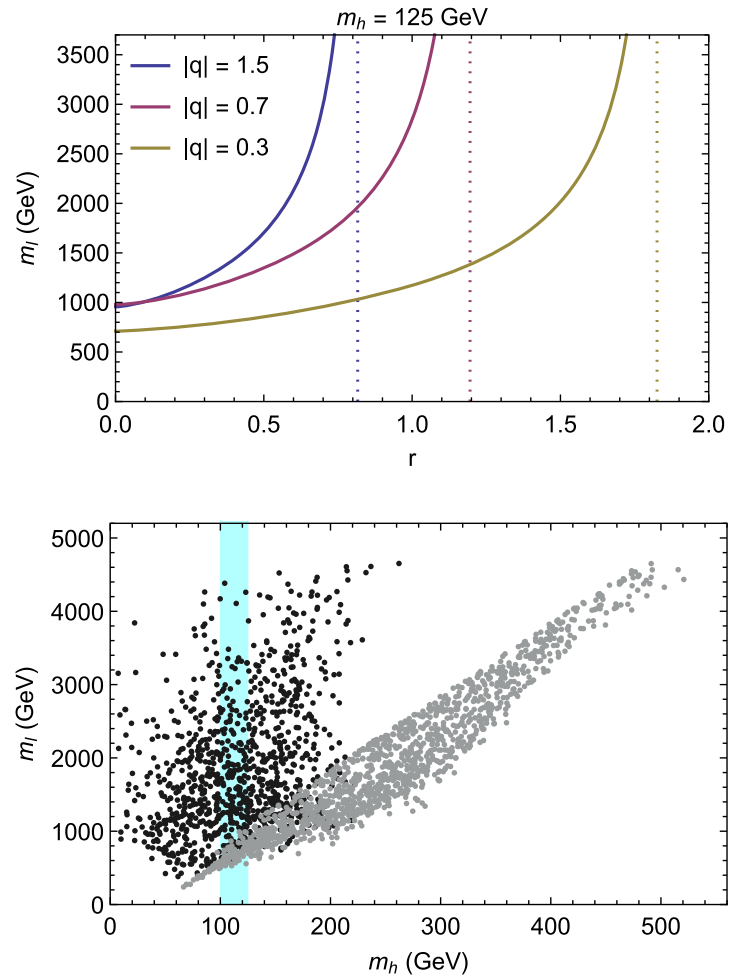

FIG. 1. Top: The mass of the lightest top partner for different values of $|q|$ as a function of $r=\tilde{m}_{T} / m_{s}$ for $m_{h}=125 \mathrm{GeV}$. The dashed vertical lines indicate the value of $r$ for which $\beta(r)$ becomes negative. The plots are unchanged for $r \rightarrow-r$. Bottom: Scatter plot of $\left(m_{h}, m_{l}\right)$ for $|r| \in(0.5,2)$ (black points) and $r=0$ (gray points). We scan in the range $\left|y_{L, R}\right| \in(0.5,2)$, $\left|\tilde{m}_{T}\right| \in(0.5,4.5) \mathrm{TeV}, q \in(-2,-0.3)$ and $\left|\delta_{1,2}\right| \in(0,2) \mathrm{TeV}$. 
$150 \mathrm{GeV}$. The prediction of the $\mathrm{MCHM}_{5}$ corresponds to the value at $r=0$. As we can see, the mass of the lightest state always increases with $r$. The dotted vertical lines signal the point $q^{2} r^{4} \rightarrow 1$, corresponding to $\beta$ (and thus the quartic) crossing zero, see Eq. (11). At that point, while the Higgs boson remains light, $m_{l}$ becomes basically unbounded. This is, however, never realized exactly in a realistic numerical scan. Points with $q^{2} r^{4}>1$ are discarded from the analysis, because leading to a negative $\beta$, as can be inspected from Eq. (11). On the other hand, it is important to notice that $m_{l}$ is significantly larger than in the $\mathrm{MCHM}_{5}$ even for $r$ and $q$ far from $q^{2} r^{4}=1$. Thus, heavier top partners do not correspond to fine-tuning the new free parameter $r$ to a very specific value but rather to a general prediction of the soft breaking setup. Note that this behavior is in qualitative contrast to the original $\mathrm{MCHM}_{5}$, where $\beta$ becomes small as $q \rightarrow 1$, namely, when $S O(5)_{R}$ gets restored, which would, however, lead to a vanishing top mass.

The results are confirmed numerically by the plot shown in the bottom panel of Fig. 1, where $\left(m_{h}, m_{l}\right)$ are obtained from a numerical scan, using the exact expression for $\beta$. The window $m_{h} \in(100,125) \mathrm{GeV}$, visualized by the blue stripe, is considered to take into account running effects to the scale $f_{\pi}$. The effect of the singlet $s$ can be seen as effectively reducing the Higgs mass which is consistent with a certain value of $m_{l}$, compared to the $\mathrm{MCHM}_{5}$ (depicted by gray points). For instance, $m_{l} \simeq 3 \mathrm{TeV}$ is compatible with $m_{h} \approx 100 \mathrm{GeV}$ in the $\mathrm{sMCHM}_{5}$, whereas that would require $m_{h} \approx 350 \mathrm{GeV}$ in the $\mathrm{MCHM}_{5}$. This is just an equivalent way of looking at Eq. (12), where the ratio $\beta\left(r^{2}\right) / \beta(0)=m_{h}^{2}\left(r^{2}\right) / m_{h}^{2}(0)<1$ gives the correct estimate for the compression of the Higgs mass. Of course, a top partner around $3 \mathrm{TeV}$ is also compatible with the correct Higgs mass in the $\mathrm{MCHM}_{5}$, but that would come inevitably with a raise in $f_{\pi}$ and, consequently, in the irreducible tuning. A more comprehensive analysis is left for future work.

General case.-We finally discuss the results for the case in which all the elementary vectorlike fermions are active below the condensation scale. To this end, we derived an expression for $\beta$ following the procedure of the previous subsection, setting $m_{v}=m_{w} \equiv m_{d}$ for simplicity, which we refer to as $\beta_{f}$ in the following. The top mass is still well approximated by Eq. (11).

In addition to $q$ and $r$, we introduce the elementary doublet-to-singlet ratio $x=m_{d} / m_{s}$. The mass of the lightest state $m_{l}$ is now estimated as

$$
\begin{aligned}
m_{l}^{2} & \simeq \min \left\{m_{Q}^{2}, \tilde{m}_{T}^{2}, m_{s}^{2}, m_{d}^{2}\right\} \\
& =m_{Q}^{2} \times \min \left\{1,1 / q^{2}, 1 / q^{2} r^{2}, x^{2} / q^{2} r^{2}\right\},
\end{aligned}
$$

where $m_{Q}$ obeys Eq. (13) with $\beta$ replaced by $\beta_{f}$. We have checked that $\beta_{f}$ approaches zero for $r \rightarrow \infty$ at fixed $x$, corresponding to the case of massless elementary fermions where no potential is generated.

We identify three different regions depending on $x$. For $|x| \lesssim 2, m_{l}$ can be at most lifted to $\approx 2 \mathrm{TeV}$, while for $|x| \gtrsim 4$, the results of the singlet case are recovered. As for the intermediate region, the estimate based on $\beta_{f}$ is shown in the upper panel of Fig. 2 for $x=2.7$ and $f_{\pi} \approx 800 \mathrm{GeV}$. The knees signal where $s$ becomes the lightest state. As before, dotted lines correspond to a vanishing $\beta_{f}$, which is never realized in a realistic scan. Nevertheless, $m_{l}$ can be generically well above $2 \mathrm{TeV}$. In the bottom panel of Fig. 2 we show $\left(m_{h}, m_{l}\right)$ obtained via a numerical scan. As we can see, the result is qualitatively the same as in Fig. 1, while the region with heavy partners is more densely populated in the blue band compatible with the Higgs mass. (We have checked that restoring the global symmetry in the linear mixings is in fact crucial for lifting the top partners, by allowing for an arbitrary mixing of the new fields filling the multiplets with the resonances and finding that the point where the symmetry is restored leads to the largest gain.)
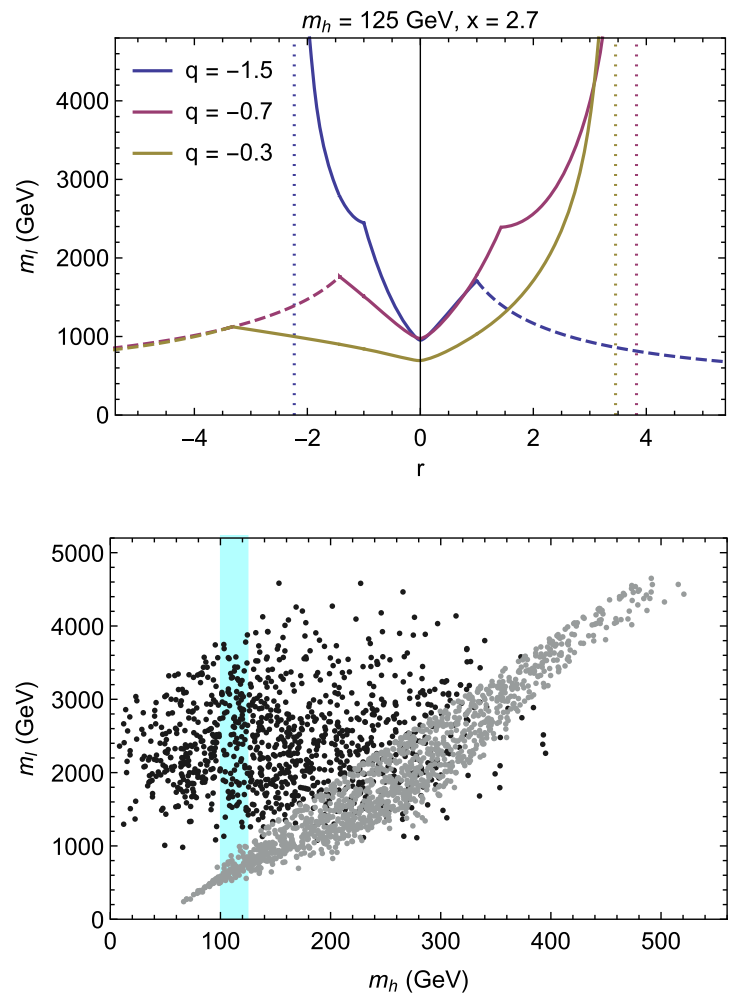

FIG. 2. Top: The mass of the lightest top partner for different values of $q$ as a function of $r$ with $m_{h}=125 \mathrm{GeV}$ and $x=2.7$. The vertical dotted lines indicate $r$ such that $\beta_{f}=0$. Dashed curves show configurations for which a decrease in $m_{l}$ is driven by the lightness of the new singlet. Bottom: The mass of the lightest top partner, $m_{l}$, as a function of $m_{h}$ in the $\mathrm{sMCHM}_{5}$ (black points) and in the $\mathrm{MCHM}_{5}$ (gray points). The scan for the $\mathrm{sMCHM}_{5}$ assumes $\left|y_{L, R}\right| \in(1,2), q \in(-2,-0.3),\left|\tilde{m}_{T}\right| \in(5,10) \mathrm{TeV},|r| \in(1.5,5)$ and $\left|\delta_{1,2}\right| \in(0,2) \mathrm{TeV}$. The doublet masses $m_{w, v}$ are scanned independently in the range $\left|m_{w, v} / m_{s}\right| \in(2,4)$. 
Since the largest $m_{l}$ is typically found above the knee, heavy top partners favors the case of the singlet $s$ as the lightest particle. This implies that the spectrum can be stabilized without requiring the spin- $1 / 2$ resonances to lie much below the naive cutoff of the strong dynamics, as needed in the conventional case to reproduce the correct Higgs mass.

Conclusions. - In this Letter, we have proposed a new way of breaking the Goldstone symmetry in $\mathrm{CH}$ scenarios, which is responsible for a nonvanishing Higgs potential. Instead of violating it via assuming the (SM-like) fermions not to fill complete representations of the global symmetry $[S O(5)$ in our case], we break it "softly," i.e., via finite vectorlike masses lifting the additional degrees of freedom that fill the representations beyond direct LHC reach. As we have shown explicitly for a two-side incarnation, this allows us to reduce the amount of Goldstone-symmetry breaking such that for fixed $f_{\pi}$ the large top mass can be reproduced without the necessity of vastly lowering the masses of the lightest top partners, the latter option starting to be in tension with LHC searches. For example, for $f_{\pi} \approx$ $800 \mathrm{GeV}$ it is possible to lift the lightest partners from $m_{l} \lesssim 1$ up to $m_{l} \sim 4 \mathrm{TeV}$, coming closer to the general scale of $\mathrm{CH}$ resonances. While the light top partners might thus be hard to detect directly at the LHC (and are generically above current LHC limits), for this setup they would be fully discoverable at the FCC. In this context, we finally note that a further phenomenological survey of the proposed SMCHM would be interesting, including an analysis of electroweak precision observables, Higgs physics, and direct searches for (elementary and composite) fermionic resonances, which is however beyond the scope of the present Letter.

We are grateful to Roberto Contino and Tommi Alanne for useful discussions.

*simone.blasi@mpi-hd.mpg.de

†fgoertz@mpi-hd.mpg.de
[1] D. B. Kaplan and H. Georgi, $\mathrm{SU}(2) \times \mathrm{U}(1)$ breaking by vacuum misalignment, Phys. Lett. 136B, 183 (1984).

[2] D. B. Kaplan, H. Georgi, and S. Dimopoulos, Composite Higgs scalars, Phys. Lett. 136B, 187 (1984).

[3] M. J. Dugan, H. Georgi, and D. B. Kaplan, Anatomy of a composite Higgs model, Nucl. Phys. B254, 299 (1985).

[4] D. B. Kaplan, Flavor at SSC energies: A new mechanism for dynamically generated fermion masses, Nucl. Phys. B365, 259 (1991).

[5] K. Agashe, R. Contino, and A. Pomarol, The minimal composite Higgs model, Nucl. Phys. B719, 165 (2005).

[6] R. Contino, Y. Nomura, and A. Pomarol, Higgs as a holographic pseudo Goldstone boson, Nucl. Phys. B671, 148 (2003).

[7] R. Contino, L. Da Rold, and A. Pomarol, Light custodians in natural composite Higgs models, Phys. Rev. D 75, 055014 (2007).

[8] O. Matsedonskyi, G. Panico, and A. Wulzer, Light top partners for a light composite Higgs, J. High Energy Phys. 01 (2013) 164.

[9] A. Pomarol and F. Riva, The composite Higgs and light resonance connection, J. High Energy Phys. 08 (2012) 135.

[10] C. Csaki, A. Falkowski, and A. Weiler, The flavor of the composite pseudo-Goldstone Higgs, J. High Energy Phys. 09 (2008) 008.

[11] S. De Curtis, M. Redi, and A. Tesi, The 4D composite Higgs, J. High Energy Phys. 04 (2012) 042.

[12] F. Goertz, Composite Higgs theory, Proc. Sci., ALPS2018 (2018) 012.

[13] A. Carmona and F. Goertz, A naturally light Higgs without light top partners, J. High Energy Phys. 05 (2015) 002.

[14] A. Carmona and F. Goertz, Lepton Flavor and Nonuniversality from Minimal Composite Higgs Setups, Phys. Rev. Lett. 116, 251801 (2016).

[15] A. Carmona and F. Goertz, A flavor-safe composite explanation of $R_{K}$, Nucl. Part. Phys. Proc. 285-286, 93 (2017).

[16] A. Carmona and F. Goertz, Recent $\boldsymbol{B}$ physics anomalies: A first hint for compositeness? Eur. Phys. J. C 78, 979 (2018).

[17] G. Panico, M. Redi, A. Tesi, and A. Wulzer, On the tuning and the mass of the composite Higgs, J. High Energy Phys. 03 (2013) 051.

[18] G. Panico and A. Wulzer, The discrete composite Higgs model, J. High Energy Phys. 09 (2011) 135. 